\title{
Experimental Investigation of a Broadband High-Temperature Superconducting Terahertz Mixer Operating at Temperatures between 40 and $77 \mathrm{~K}$
}

\author{
Xiang Gao ${ }^{1}$, Jia Du ${ }^{1}$, Ting Zhang ${ }^{2}$, Y. Jay Guo ${ }^{2}$, Cathy P. Foley ${ }^{1}$ \\ 1 CSIRO Manufacturing, PO Box 218, Lindfield, NSW 2070, Australia. \\ 2 Global Big Data Technologies Centre, University of Technology, Sydney, Ultimo, NSW 2007, \\ Australia. \\ Corresponding author: Xiang.Gao@csiro.au
}

\begin{abstract}
This paper presents a systematic investigation of a broadband thin-film antenna-coupled high-temperature superconducting (HTS) terahertz (THz) harmonic mixer at relatively high operating temperature from 40 to $77 \mathrm{~K}$. The mixer device chip was fabricated using the CSIRO established step-edge $\mathrm{YBa}_{2} \mathrm{Cu}_{3} \mathrm{O}_{7-\mathrm{x}}$ (YBCO) Josephson junction technology, packaged in a well-designed module and cooled in a temperature adjustable cryocooler. Detailed experimental characterizations were carried out for the broadband HTS mixer at both the $200 \mathrm{GHz}$ and $600 \mathrm{GHz}$ bands in harmonic mixing mode. The DC current-voltage characteristics (IVCs), bias current condition, local oscillator (LO) power requirement, frequency response as well as conversion efficiency under different bath temperatures were thoroughly investigated for demonstrating the frequency down-conversion performance.
\end{abstract}

Keywords Terahertz, harmonic mixer, high-temperature superconducting Josephson junction, broadband mixer

\section{Introduction}

Terahertz (THz) waves have many unique properties that can potentially be applied in many areas such as astronomical observation, nondestructive testing, security screening, medical imaging and spectroscopy [1]. Compared with infrared and microwaves, $\mathrm{THz}$ wave combines the merits of good penetrating capability and high spatial resolution, which accordingly offers a promising alternative solution for remote sensing [2] applications. In addition, abundant bandwidth resource at $\mathrm{THz}$ band is beneficial to facilitating the implement of ultrahigh bitrate wireless communication link [3] for accommodating the drastically increasing demand in various electronics scenarios.

In order to exploit the potential of $\mathrm{THz}$ waves, the significant challenge imposed by atmospheric absorption attenuation has to be overcome, especially for $\mathrm{THz}$ remote sensing and wireless communication. Accordingly, ultrasensitive heterodyne receiver frontends are desired for those $\mathrm{THz}$ sensing or wireless systems. Superconducting mixer devices are promising components for $\mathrm{THz}$ receiver frontends considering their superior sensitivity and low local oscillator (LO) power requirement. Generally, they are divided into low-temperature superconducting (LTS) and high-temperature superconducting (HTS) mixers based on the critical temperature of the superconducting materials. Typical LTS devices like superconductor-insulator-superconductor (SIS) mixers [4]-[6] and hot electron bolometer (HEB) mixers [7]-[9] are more sensitive than the HTS mixers, but they operate at liquid helium temperature $(4.2 \mathrm{~K})$ range thus requiring expensive and bulky cryogenic facilities. In comparison, the HTS devices operate at higher bath temperatures, where cheaper and portable single-stage cryocoolers can be used. In addition, the HTS mixers can work at higher THz frequencies than that of the SIS mixers (with upper limitation of around $1 \mathrm{THz}$ ) benefitting from their larger superconducting energy 
gap. They also can have potentially wider intermediate-frequency (IF) bandwidth than that of the LTS HEB mixers due to faster intrinsic instantaneous response although in practice the IF bandwidth is often restricted by the bandwidth of the readout electronics (e.g. IF amplifier).

In spite of so many attractive features, research development on HTS THz mixers lags behind due to the difficulty in fabricating reliable and reproducible HTS Josephson junctions with suitable parameters required for high performance mixers. So far, there have been very few demonstrations of HTS Josephson-junction mixers at THz band reported in literature [10]-[13]. These reported HTS mixers, however, mostly operated at much lower temperatures than $77 \mathrm{~K}$, which still relied on large and expensive cryogenic facilities thus resulting in high system cost. Furthermore, the reported measurement results did not discuss the influence of bath temperature on the mixer operation conditions and conversion performance. Based on CSIRO well-established HTS step-edge junction technology [14] and previous achievements in developing various HTS passive and active devices at high frequencies [15]-[19], we have, in this work, developed a broadband HTS Josephson-junction THz harmonic mixer. The experimental characterization results of the broadband HTS THz mixer at two THz bands (200 GHz and $600 \mathrm{GHz}$ ) and at bath temperature from $40 \mathrm{~K}$ to $77 \mathrm{~K}$ are presented in this paper.

\section{Mixer Description and Characterization Set-up}

The thin-film antenna-coupled HTS THz mixer chip was designed and implemented on a 0.5-mm thick $\mathrm{MgO}$ substrate. Fig. 1 shows the photographs of the fabricated device where a broadband log-periodic circular-toothed thin-film antenna couples the THz signal into a Josephson junction located between the two wings at the center. The antenna features a self-complementary structure with log-periodic scaling factor of 0.6, whose largest and smallest outer arm radius are around $227.7 \mu \mathrm{m}$ and $29.5 \mu \mathrm{m}$ respectively, resulting in a very wide operating bandwidth covering the frequencies from $200 \mathrm{GHz}$ to $600 \mathrm{GHz}$. Despite that the average input impedance is around 75-80 $\Omega$, the mismatch between the $\mathrm{THz}$ antenna and Josephson junction has been minimized at $200 \mathrm{GHz}$ and $600 \mathrm{GHz}$ bands by taking advantage of the frequency dependent impedance fluctuation characteristics. The antenna radiates a linearly polarized wave with the polarization direction shown in Fig. 1. On the other hand, a coplanar waveguide (CPW) line was designed to optimize the power transmission of the LO and intermediate-frequency (IF) signals. Benefitting from the radiation mechanism of the log-periodic antenna, there is no leakage of $\mathrm{THz}$ electric current thus removing the need of any isolation network like choke filter. The details of the electromagnetic design and analysis of the antenna and microwave coupling circuit are to be reported elsewhere. Fig. 1(b) shows the enlarged view of the HTS step-edge Josephson junction, which is a 2- $\mu \mathrm{m}$ wide YBCO strip across the step pattern created by using a standard photolithography and Ar-ion beam etching techniques [14]. Finally, the fabricated HTS mixer chip was packaged along with a bias-tee network into a specially designed housing as a THz mixer module, as shown in the inset of Fig. 2. Here, two mixer devices were fabricated on a $10 \times 10-\mathrm{mm}^{2} \mathrm{MgO}$ substrate, and two 3-mm diameter hemispherical Silicon $(\mathrm{Si})$ lens were attached on the back of the substrate for enhancing the coupling of $\mathrm{THz}$ radiation. 


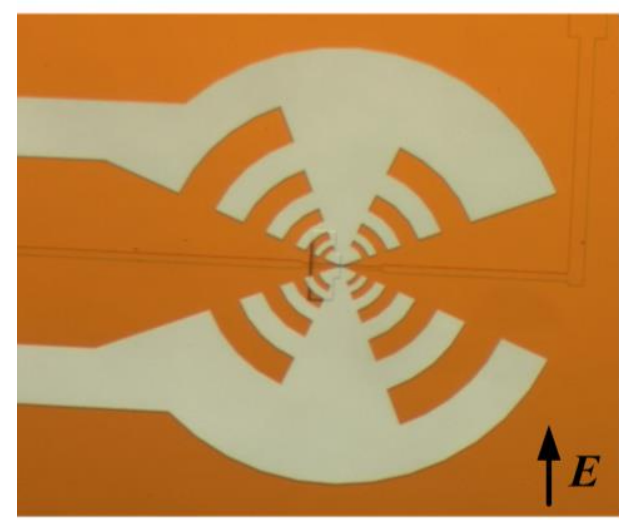

(a)

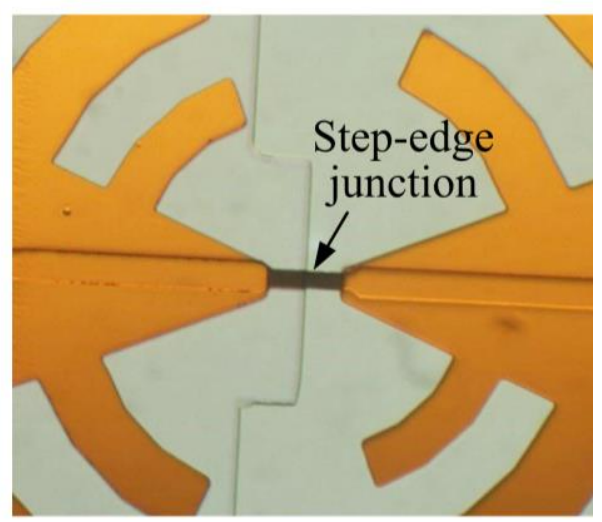

(b)

Fig. 1. Micrograph showing (a) the log-periodic thin-film antenna and CPW transmission line on a fabricated THz mixer chip and (b) an enlarged view of the HTS step-edge Josephson junction.

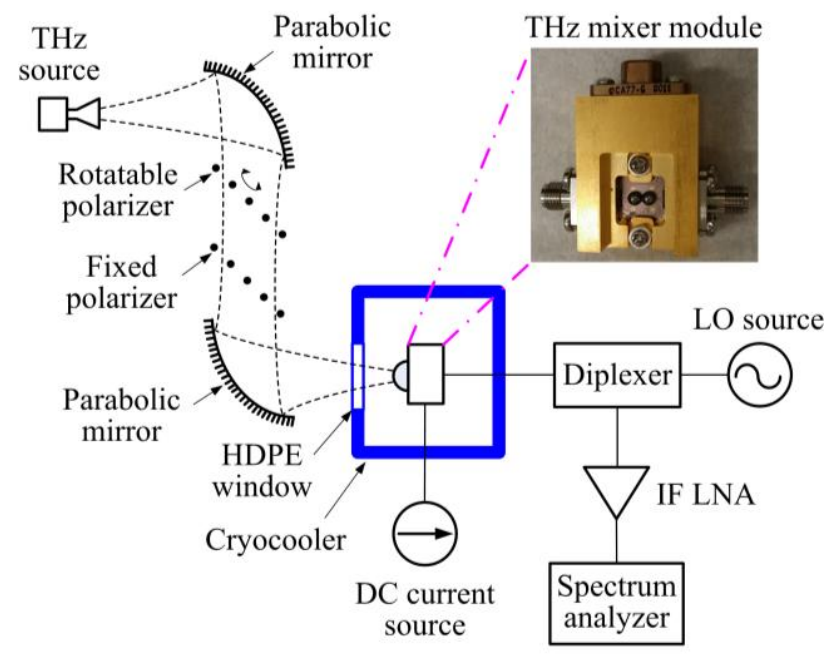

Fig. 2. The schematic diagram of characterization set-up for the HTS Josephson-junction THz harmonic mixer. Inset is a photograph showing the packaged $\mathrm{THz}$ mixer module.

Fig. 2 shows the schematic diagram of the experimental set-up for characterizing the HTS Josephson-junction $\mathrm{THz}$ harmonic mixer. The packaged $\mathrm{THz}$ mixer module was mounted in a temperature adjustable cryocooler with the $\mathrm{Si}$ lens facing the window. The $\mathrm{THz}$ radiation was generated from a commercial solid-state source $(200 \mathrm{GHz}$ or $600 \mathrm{GHz}$ Active Multiplier Chains) and propagates along the quasi-optics link to the mixer module. The mixer was pumped by an LO signal from a Wiltron 68075B generator. Under an appropriate DC bias condition the heterodyne mixing occurs between the THz signal and the high-order harmonics of the LO signal. The down-converted IF output signal, which shares the same port of the mixer module with the LO signal via a diplexer for isolation, was amplified and then recorded by an Agilent E4407B spectrum analyzer.

In order to have the HTS THz mixer operating within its linearity region, two wire-grid polarizers (one rotatable and the other fixed) were utilized to regulate the THz power. The polarizers were placed in $45^{\circ}$ tilted orientations for preventing multiple reflections between them. The theoretical formula for $\mathrm{THz}$ power attenuation $L_{\mathrm{att}}$ and polarizer rotation angle $\theta$ can be described as [20]

$$
L_{\text {att }}=1 / \sin ^{4}[\arctan (\sqrt{2} \cot \theta / 2)]
$$

where $\theta=0^{\circ}$ is corresponding to the case of perfect polarization matching between the $\mathrm{THz}$ wave and wire-grid polarizers. However, considering the non-ideal polarization performance of $\mathrm{THz}$ wire-grids as 
well as possible alignment errors from the quasi-optics set-up, we measured the actual relationship of $L_{\text {att }}$ vs $\theta$, and compared with the theoretical calculation (Eq. (1)) as shown in Fig. 3. The measured results deviate slightly from the theoretical relationship for larger polarizer rotation angles. The calibration of the THz power attenuation was carried out by using a VDI Schottky detector placed at the focus of second parabolic mirror in Fig. 2 (i.e. the same position as that of the THz mixer module). On the whole, all the curves in Fig. 3 have consistent trends and, those measured values can be used for the estimation of $\mathrm{THz}$ power coupled into the junction which will be discussed in Section 3.

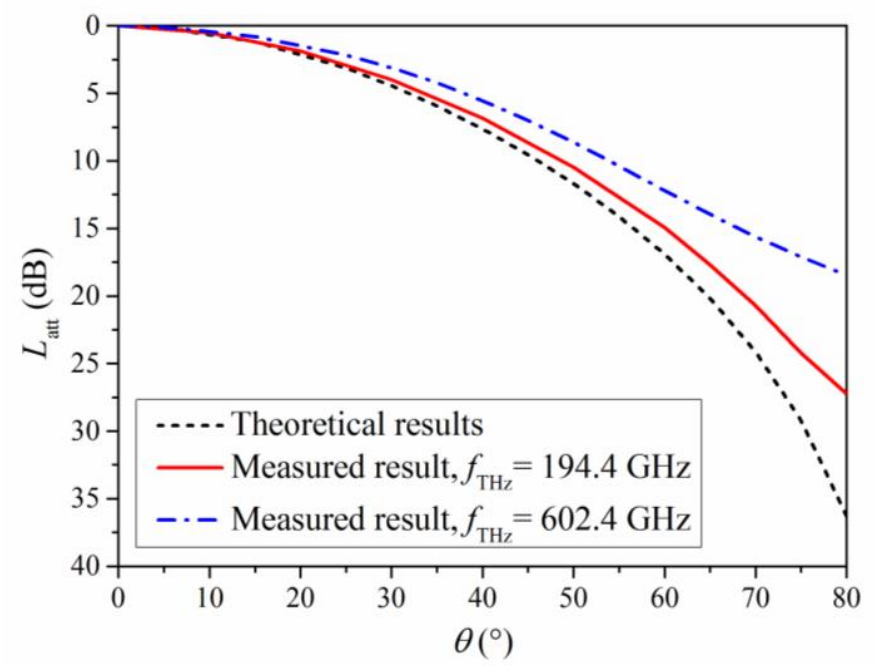

Fig. 3. Theoretical and measured results of the THz power attenuation $L_{\text {att }}$ versus polarizer rotation angle $\theta$.

\section{Measurement Results and Discussion}

\subsection{Characteristics and Coupled Power Estimation}

Fig. 4(a) shows the measured DC current-voltage characteristics (IVCs) of the HTS THz mixer at bath temperature $T$ from $40 \mathrm{~K}$ to $77 \mathrm{~K}$. The step-edge Josephson junction has a normal resistance $R_{\mathrm{n}}$ of around $5 \Omega$, and its critical current $I_{\mathrm{c}}$ decreases from about $310 \mu \mathrm{A}$ at $40 \mathrm{~K}$ to $15 \mu \mathrm{A}$ at $77 \mathrm{~K}$ (shown in the inset). Under the illumination of THz signals, as clearly shown in Fig. 4(b) and 4(c), the junction exhibits different IVCs with the $I_{\mathrm{c}}$ partly suppressed and a series of Shapiro steps induced at the voltages $V_{n}=$ $n \Phi_{0} f_{\mathrm{THz}}$ (where $n$ is an integer, $\Phi_{0}$ is the magnetic flux quantum, and $f_{\mathrm{THz}}$ is the THz signal frequency). As predicted by the Josephson voltage-frequency relationship, the voltage of the induced first Shapiro step is located exactly at $0.402 \mathrm{mV}$ for $f_{\mathrm{THz}}=194.4 \mathrm{GHz}$ and $1.246 \mathrm{mV}$ for $f_{\mathrm{THz}}=602.4 \mathrm{GHz}$, respectively. These results showed that the THz signal is effectively coupled into the step-edge Josephson junction through the Si lens and log-periodic thin-film antenna. Fig. 4(d) shows the measured IVCs at temperature of $40 \mathrm{~K}$ when the mixer is pumped by different power levels of $\mathrm{THz}$ radiation at $602.4 \mathrm{GHz}$. The variation of the $\mathrm{THz}$ power level was achieved by manually rotating the first wire-grid polarizer to specific angles (the relationship is shown in Fig. 3). It can be clearly seen that, the $I_{\mathrm{c}}$ suppression and Shapiro current step heights increase as the applied $\mathrm{THz}$ signal power rises. 


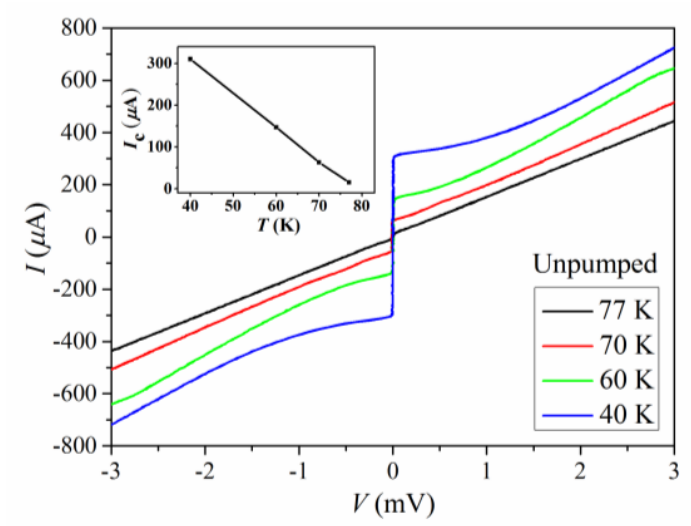

(a)

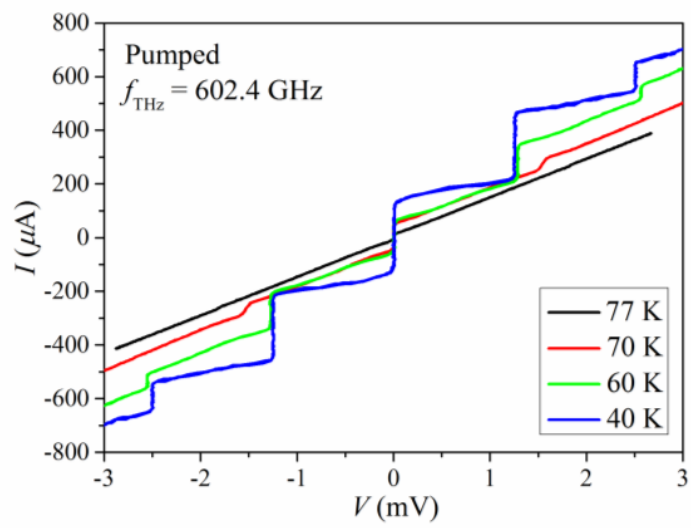

(c)

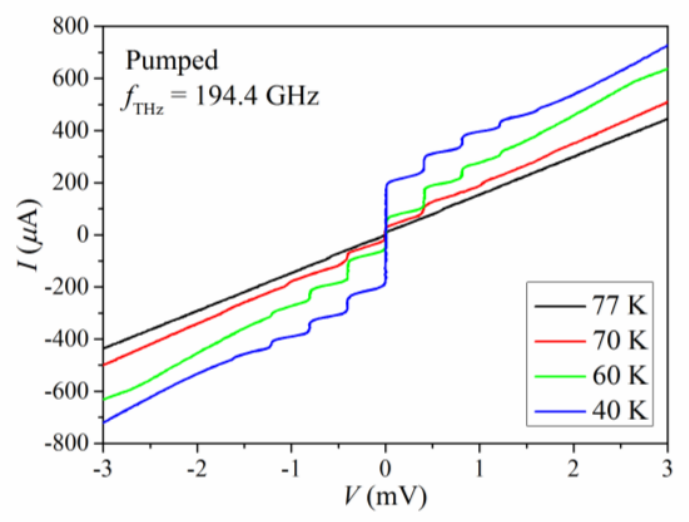

(b)

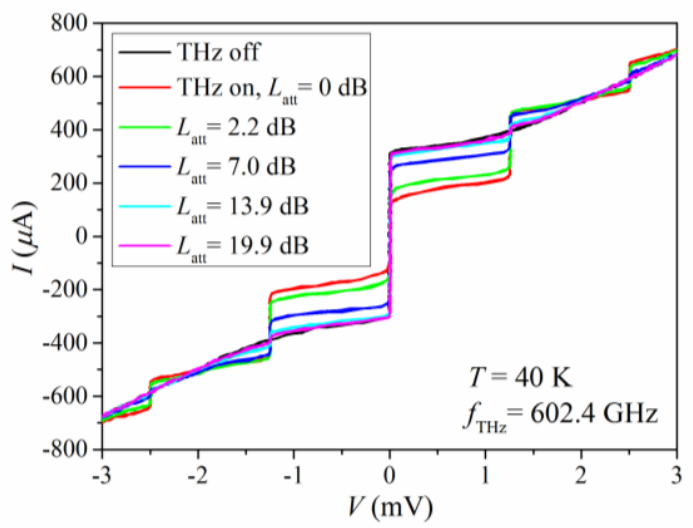

(d)

Fig. 4. DC IVCs of the HTS THz mixer: (a-c) at different bath temperatures when (a) unpumped, and pumped with THz signals of (b) $194.4 \mathrm{GHz}$ and (c) $602.4 \mathrm{GHz}$; (d) under different power levels of THz radiation at $602.4 \mathrm{GHz}$ and $40 \mathrm{~K}$.

Based on the measured IVCs, a Bessel-function fitting is applied to estimate the THz power coupled into the Josephson junction $P_{\mathrm{THz}}$. Theoretically, the $P_{\mathrm{THz}}$ can be estimated from the reduced critical current $I_{\mathrm{c}}^{\prime}$ and their relationship can be expressed as [21]

$$
I_{\mathrm{c}}^{\prime}=I_{\mathrm{c}} J_{0}\left[\sqrt{2 P_{\mathrm{THz}} R_{\mathrm{n}}} /\left(\Phi_{0} f_{\mathrm{THz}}\right)\right]
$$

where $J_{0}$ is the zeroth order Bessel function of the first kind. Although the $P_{\mathrm{THz}}$ can be determined directly from Eq. (2) for a given $I_{\mathrm{c}}^{\prime}$ value, there might exist the measurement and reading errors for single pumped current-voltage curve. Hence, an improved approach based on the combination of multiple pumped curves with different values of $I_{\mathrm{c}}^{\prime}$ is used to obtain more accurate result. Fig. 5(a) and 5(b) show the measured $I_{\mathrm{c}}^{\prime}$ versus $\mathrm{THz}$ power attenuation $L_{\text {att }}$ for $\mathrm{THz}$ radiations at $194.4 \mathrm{GHz}$ and $602.4 \mathrm{GHz}$ signals, respectively. The THz power attenuation $L_{\text {att }}$ was converted from the wire-grid polarization angle $\theta$ using the experimental relationship shown in Fig. 3. By fitting the measured results with a Bessel function of

$$
I_{\mathrm{c}}^{\prime}=I_{\mathrm{c}} J_{0}\left(\alpha / \sqrt{L_{\mathrm{att}}}\right)
$$

and comparing the Eq. (3) with Eq. (2), one can obtain the $P_{\mathrm{THz}}$ as

$$
P_{\mathrm{THz}}=\alpha^{2} \Phi_{0}^{2} f_{\mathrm{THz}}^{2} /\left(2 R_{\mathrm{n}} L_{\mathrm{att}}\right)
$$

Using this new method, the THz signal power coupled into the junction without attenuation ( $L_{\text {att }}=0 \mathrm{~dB}$ ) was estimated as $-45.4 \mathrm{dBm}$ for $194.4 \mathrm{GHz}$ and $-33.5 \mathrm{dBm}$ for $602.4 \mathrm{GHz}$ signal, respectively. 


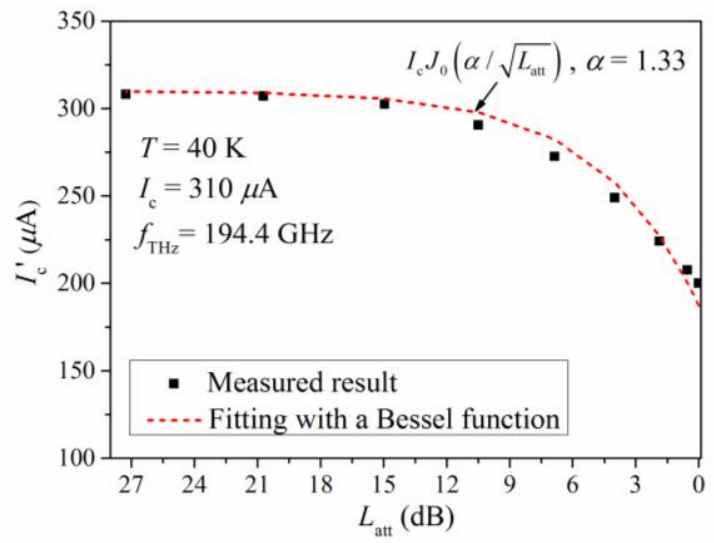

(a)

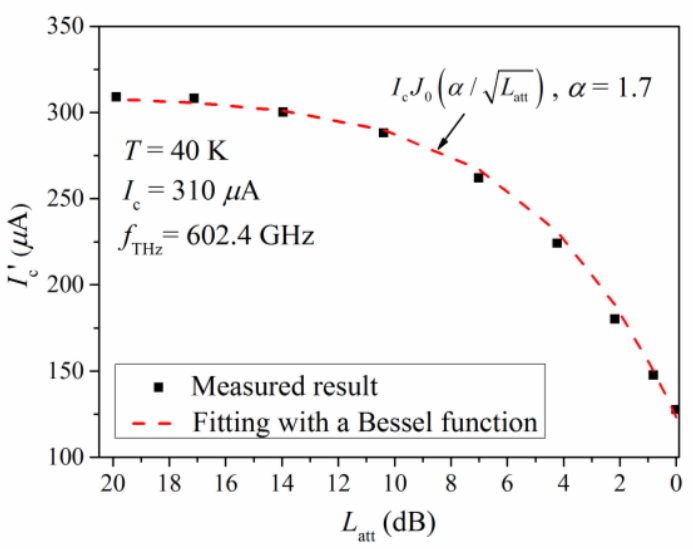

(b)

Fig. 5. The relationship of reduced critical current $I_{\mathrm{c}}{ }^{\prime}$ with THz power attenuation $L_{\text {att }}$ for (a) $194.4 \mathrm{GHz}$ and (b) $602.4 \mathrm{GHz}$ signals.

\subsection{Harmonic Mixing Performance}

The frequency down-conversion performance of the HTS THz harmonic mixer was characterized in detail. Firstly, the bias current condition was examined. Fig. 6 shows the measured IF output power, $P_{\mathrm{IF}}$, versus bias current, $I_{\mathrm{B}}$, for different $\mathrm{LO}$ power, $P_{\mathrm{LO}}$, at temperature of $40 \mathrm{~K}$. Fig. 6(a) and 6(b) are measured results for $\mathrm{THz}$ input signal at frequencies of $194.4 \mathrm{GHz}$ and $602.4 \mathrm{GHz}$ respectively, thus corresponding to 6th and 20th harmonic mixing under a similar LO frequency of $\sim 30 \mathrm{GHz}$. As clearly shown in Fig. 6, the HTS Josephson-junction mixer has relatively wide bias current ranges (more than $200 \mu \mathrm{A}$ at $P_{\mathrm{LO}}=-31 \mathrm{dBm}$ ) for both $\mathrm{THz}$ bands, but the range reduces with decreasing the LO power. The phenomenon of LO power dependence of the $P_{\mathrm{IF}} \mathrm{Vs} I_{\mathrm{B}}$ relationship can be explained from the variations of DC IVC under different values of $P_{\mathrm{LO}}$. As shown in Fig. 7, the lower limit of $I_{\mathrm{B}}$ basically depends on the suppressed junction critical current which is smaller for higher LO power, while the upper limit lies in a certain point before the Josephson junction entering into the normal state. The junction moves into its normal state at a higher $I$ point under higher LO pumping power $P_{\mathrm{LO}}$. The same LO power levels as that in Fig. 6 were used for the IVC plotted in Fig. 7 and it shows that the operation bias current range reduces with decreasing the LO pumping power. The $P_{\mathrm{IF}}$ vs $I_{\mathrm{B}}$ relationships shown in Fig. 6 exhibit strong modulation phenomenon with a number of resonant peaks appear. It appears that the number of the peaks is related to the number of harmonics of $f_{\mathrm{LO}}$; a higher harmonic order results in more resonant peaks. The experimental results showed six major peaks for the 6th order harmonic mixing at the $200 \mathrm{GHz}$ band (Fig 6a) but less than 20 peaks for the 20th order $600 \mathrm{GHz}$ band (Fig. 6b). Based on the experimental observation and the knowledge that the IF output peak usually occurs on the middle points of the IVC steps induced by the LO frequency, a plausible hypothesis is that $P_{\mathrm{IF}}$ minima do occur at integer multiples or harmonics of $f_{\mathrm{LO}}$, but not at every value. Further work is underway to investigate the mechanism and theoretically model this behavior.

Fig. 8(a) and 8(b) show the temperature dependence of the $P_{\mathrm{IF}}$ vs $I_{\mathrm{B}}$ relationship for THz radiations at 194.4 GHz and $602.4 \mathrm{GHz}$, respectively. It can be clearly seen that for both bands, the bias current ranges move downward with increasing the operating temperature from $40 \mathrm{~K}$ to $70 \mathrm{~K}$. This can be explained from the fact that the junction critical current decreases as the bath temperature rises (Fig. 4 (a)) and the optimum bias current is usually at a level slightly above the suppressed junction critical current. 


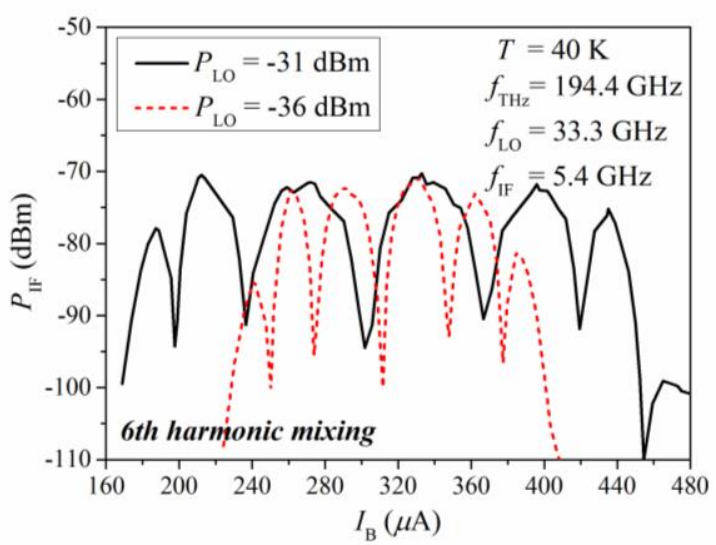

(a)

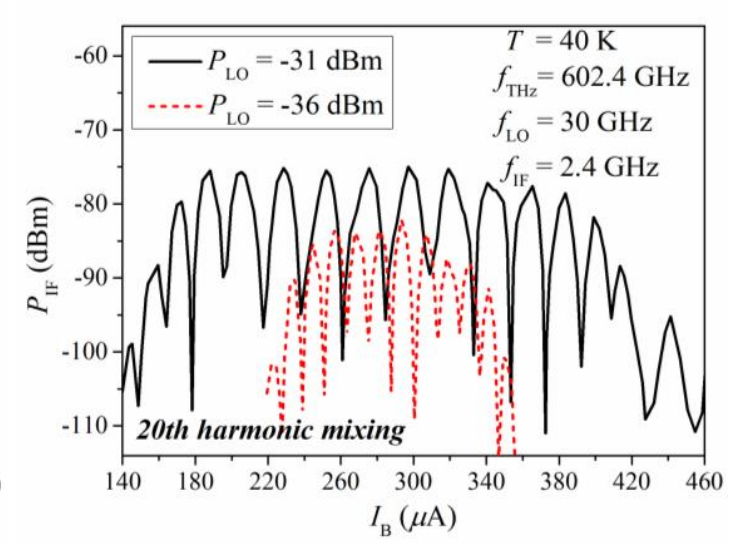

(b)

Fig. 6. IF output power $P_{\mathrm{IF}}$ versus bias current $I_{\mathrm{B}}$ for different LO pumping power $P_{\mathrm{LO}}$ : (a) $f_{\mathrm{THz}}=194.4 \mathrm{GHz}$, 6th harmonic mixing; (b) $f_{\mathrm{THz}}=602.4 \mathrm{GHz}$, 20th harmonic mixing.

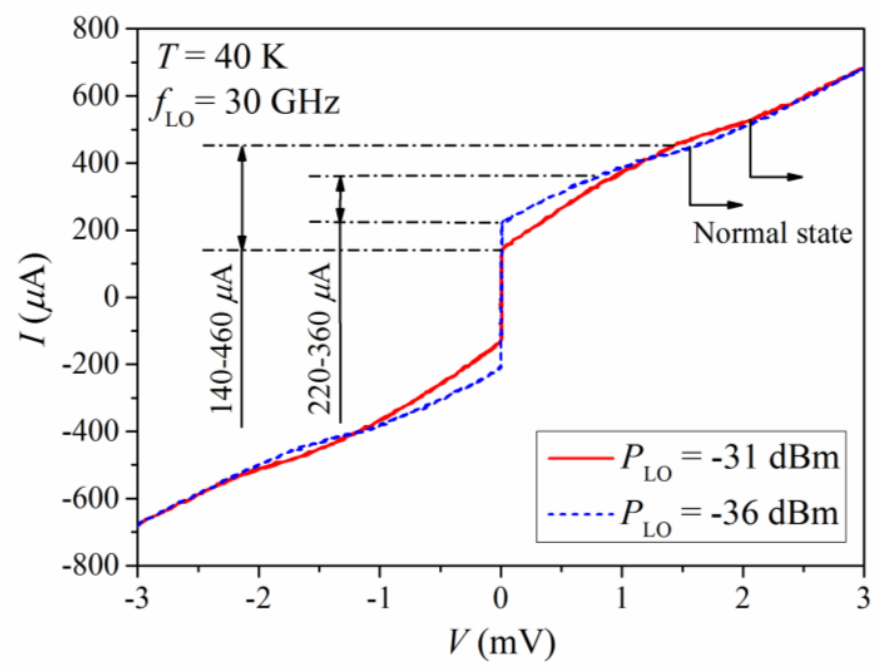

Fig. 7. DC IVCs of the HTS THz mixer under different LO pumping power $P_{\mathrm{LO}}$.

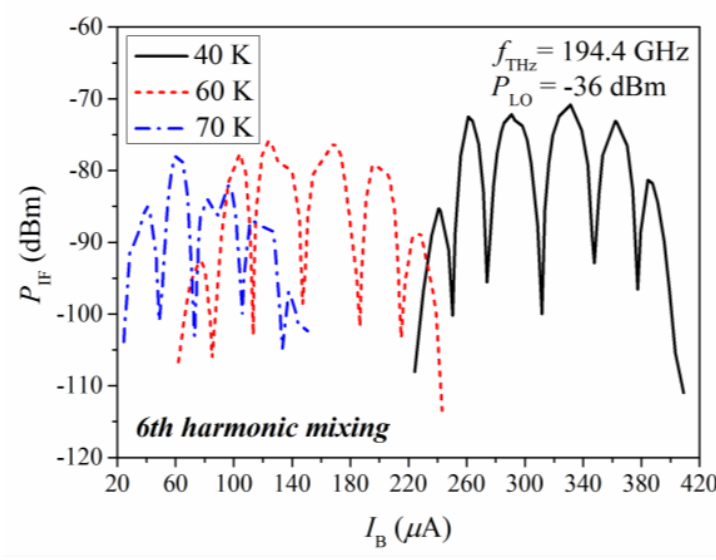

(a)

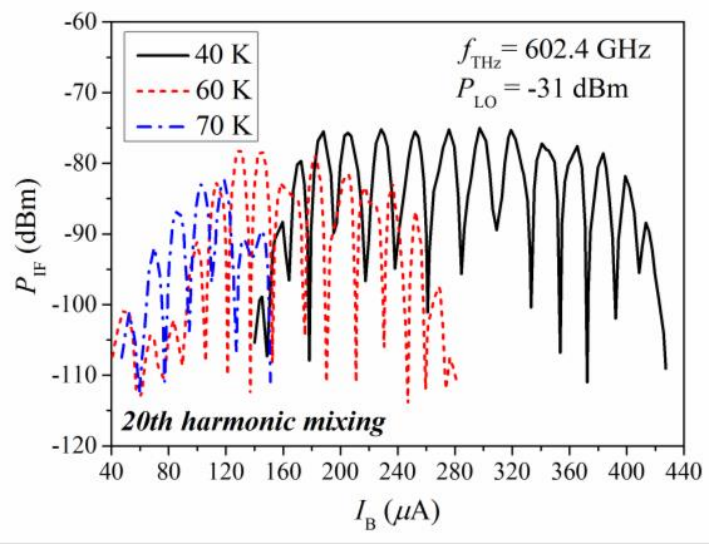

(b)

Fig. 8. IF output power $P_{\mathrm{IF}}$ versus bias current $I_{\mathrm{B}}$ for different bath temperatures: (a) $f_{\mathrm{THz}}=194.4 \mathrm{GHz}$, 6th harmonic mixing; (b) $f_{\mathrm{THz}}=602.4 \mathrm{GHz}, 20 \mathrm{th}$ harmonic mixing.

Fig. 9 shows the measured relationship of IF output power $P_{\mathrm{IF}}$ with LO pumping power $P_{\mathrm{LO}}$ at different bath temperatures, where the bias current $I_{\mathrm{B}}$ was respectively adjusted to yield maximum IF output for each temperature. The IF output varies with $\mathrm{LO}$ power but works for a wide range of $P_{\mathrm{LO}}$ 
conditions. The $P_{\mathrm{IF}}$ vs $P_{\mathrm{LO}}$ exhibits some maximum or minimum points. This behavior is more obvious at the higher operating temperature where the junction critical current is much smaller. Further theoretical study is required to understand this behavior. It can be clearly seen from Fig. 9 that, the required LO power for normal operation is only in the order of $\mu \mathrm{W}$ for both the $200 \mathrm{GHz}$ and $600 \mathrm{GHz}$ bands, much lower than that for Schottky diode mixers, which is one of the major advantages of the Josephson-junction mixer.

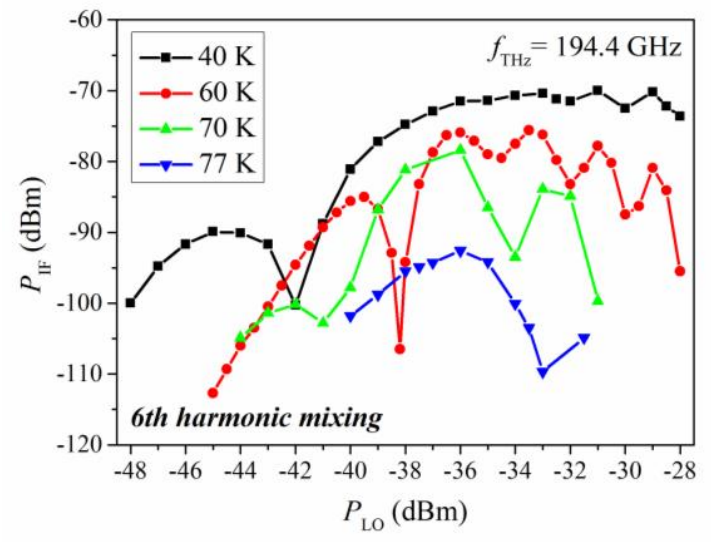

(a)

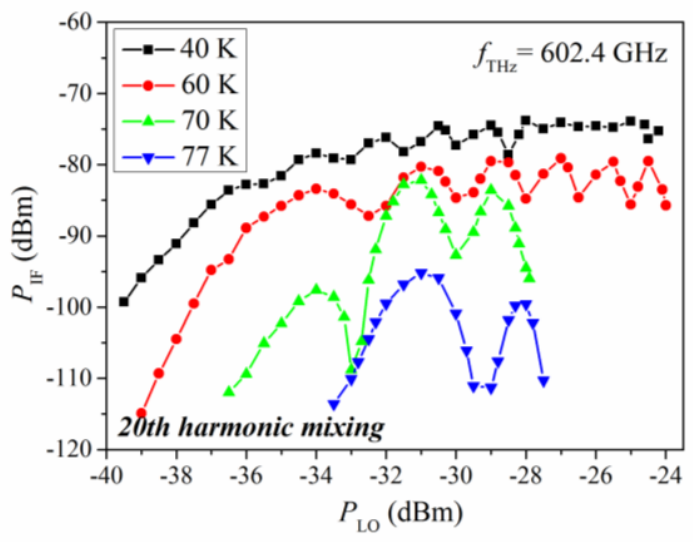

(b)

Fig. 9. IF output power $P_{\mathrm{IF}}$ versus LO pumping power $P_{\mathrm{LO}}$ at different bath temperatures: (a) $f_{\mathrm{THz}}=194.4 \mathrm{GHz}$, 6th harmonic mixing; (b) $f_{\mathrm{THz}}=602.4 \mathrm{GHz}$, 20th harmonic mixing.

The frequency response of the HTS THz harmonic mixer was characterized as shown in Fig. 10. During the measurements, the LO signal frequency was fixed as $33.3 \mathrm{GHz}$ for $200 \mathrm{GHz}$ or $30 \mathrm{GHz}$ for $600 \mathrm{GHz}$ band. Based on the 6th and 20th harmonic mixing characterizations at respective bands, the resultant IF bandwidth of the THz mixer was about $8 \mathrm{GHz}$, restricted by the operating bandwidth of the IF low noise amplifier (LNA). After calibrating the total IF link (including the LNA, diplexer and cables) and THz source power with frequency, the IF bandwidth is found to be at least $12 \mathrm{GHz}$ for either upper or lower sideband, which is relatively wide for high-bitrate communication or sensing application. The obvious dips near the center of the frequency response traces are due to the low frequency rejection characteristics of the bias-tee circuit integrated within the mixer module. The little fluctuation in those traces are believed to result from the imperfect quasi-optics link as well as measurement and calibration tolerances. In addition, it should be mentioned that based on experimental observation, the bath temperature has no any obvious influence on the mixer frequency response, accordingly only the representative result at $40 \mathrm{~K}$ was presented here. 


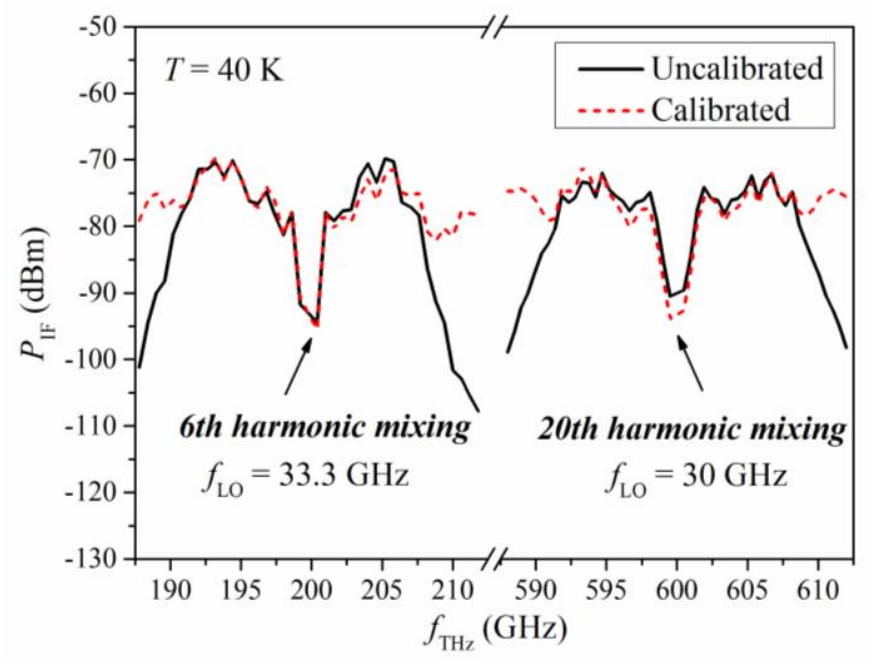

Fig. 10. Frequency response of the HTS THz mixer at $200 \mathrm{GHz}$ and $600 \mathrm{GHz}$ bands.

Fig. 11 shows the characterized conversion efficiency $G_{\text {mix }}$ versus bath temperature $T$ at both $\mathrm{THz}$ bands. The $G_{\text {mix }}$ was obtained using Eq. (5),

$$
G_{\mathrm{mix}}=P_{\mathrm{IF}} /\left(P_{\mathrm{THz}} G_{\mathrm{IF}}\right)
$$

where $P_{\mathrm{THz}}$ is the THz power coupled into Josephson junction, $P_{\mathrm{IF}}$ is the down-converted IF output power, and $G_{\mathrm{IF}}$ is the calibrated IF link gain. Here, the THz input signal was attenuated by $23.4 \mathrm{~dB}$ (194.4 $\mathrm{GHz})$ or $19.9 \mathrm{~dB}(602.4 \mathrm{GHz})$ using the wire-grid polarizer before coupled into the Junction. As shown in Fig. 10, at bath temperature of $40 \mathrm{~K}$, the $G_{\text {mix }}$ is around $-30 \mathrm{~dB}$ for $200 \mathrm{GHz}$ band and around $-50 \mathrm{~dB}$ for $600 \mathrm{GHz}$ band. This result is comparable to that of the state-of-art semiconductor even harmonic mixer (EHM) from VDI at similar frequencies [22] (at $200 \mathrm{GHz}$ bands, for example, but our mixer also operates in $600 \mathrm{GHz}$ frequency band). Up to the date, we are not aware any other reported conversion efficiency performance for HTS harmonic mixer at $\mathrm{THz}$ band. In addition, the mixer was found to operate stably over a wide temperature range up to $77 \mathrm{~K}$, i.e. the liquid nitrogen temperature. The $G_{\text {mix }}$, measured at optimum bias and LO pumping conditions, descends monotonically with the increase of bath temperature, which mainly results from the decreased dynamic resistance and junction characteristic voltage, $V_{\mathrm{c}}=I_{\mathrm{c}} R_{\mathrm{n}}$, at higher temperatures. The fact that our mixer could operate at $77 \mathrm{~K}$ is a striking result, since we are aware that the reported HTS mixers in literature so far all operated in the temperatures lower than $77 \mathrm{~K}$ ([10]-[13] for example). These results has demonstrated the superiority of the presented HTS mixer over previously reported HTS THz mixers. It is possible that the mixer conversion efficiency could be further improved through optimizing the Josephson junction parameters (e.g. higher junction characteristic voltage $V_{\mathrm{c}}$ and higher dynamic resistance $R_{\mathrm{d}}$ [21]), lowering operating temperature (thus increasing $V_{\mathrm{c}}$ and $R_{\mathrm{d}}$ values), and increasing the $\mathrm{LO}$ frequency to reduce the harmonic order. In addition, the junction-antenna impedance match may be further optimized in future designs. 


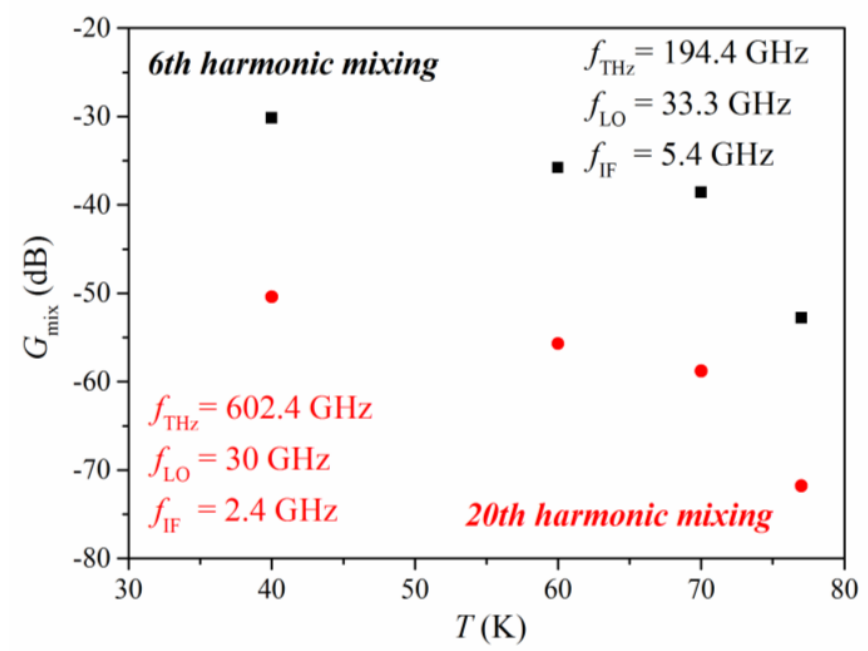

Fig. 11. Mixer conversion efficiency $G_{\text {mix }}$ versus bath temperature $T$ for $200 \mathrm{GHz}$ and $600 \mathrm{GHz}$ bands.

\section{Conclusion}

Systematic experimental investigation of a broadband antenna-coupled HTS Josephson-junction THz harmonic mixer have been presented in this paper. The mixer device was carefully designed and fabricated using the advanced step-edge YBCO junction technology established at our laboratory. The DC and RF performances of HTS mixer were thoroughly investigated at both $200-\mathrm{GHz}$ and $600-\mathrm{GHz}$ bands and at operating temperatures between $40 \mathrm{~K}$ and $77 \mathrm{~K}$, the temperature range that is attainable with a smaller and cheaper single-stage cryocooler. The presented mixer showed superior performance over those reported HTS mixers in literature at comparable frequencies and operating temperatures. In particular, the mixer could operate stably at temperature up to $77 \mathrm{~K}$, which has not been reported by any other groups to date. An IF bandwidth of over $12 \mathrm{GHz}$ was obtained for either upper or lower sideband. A conversion gain of $\sim-30 \mathrm{~dB}$ and $\sim-50 \mathrm{~dB}$ were estimated at $40 \mathrm{~K}$ for the $200 \mathrm{GHz}$ band at 6 th harmonic mixing and the $600 \mathrm{GHz}$ band at 20th harmonic mixing, respectively. The presented HTS Josephson-junction $\mathrm{THz}$ mixer will have potential applications in both $\mathrm{THz}$ wireless communication and remote sensing.

\section{Acknowledgement}

The authors would like to thank our colleagues, Dr Andrew R. Weily for the bias-tee circuit and diplexer designs, Ms Jeina Lazar for the HTS chip fabrication, and Ms Mei Shen for the mixer module packaging.

\section{References}

[1] P. H. Siegel, “Terahertz technology,” IEEE Trans. Microw. Theory Tech., vol. 50, no. 3, pp. 910-928, Mar. 2002.

[2] D. Jasteh, E. G. Hoare, M. Cherniakov, and M. Gashinova, "Experimental low-terahertz radar image analysis for automotive terrain sensing," IEEE Geosci. Remote Sens. Lett., vol. 13, no. 4, pp. 490-494, Apr. 2016.

[3] T. Schneider, "Ultrahigh-bitrate wireless data communications via THz-links; Possibilities and challenges," J. Infrared Millimeter THz Waves, vol. 36, no. 2, pp. 159-179, Feb. 2015.

[4] B.-K. Tan, G. Yassin, P. Grimes, J. Leech, K. Jacobs, and C. Groppi, "A 650 GHz unilateral finline SIS mixer fed by a multiple flare-angle smooth-walled horn,” IEEE Trans. THz. Sci. Technol., vol. 2, no. 1, pp. 40-49, Jan. 2012. 
[5] A. Karpov, D. A. Miller, J. A. Stern, B. Bumble, H. G. LeDuc, I. Mehdi, R. H. Lin, and J. Zmuidzinas, "Low noise 1 THz SIS mixer for stratospheric observatory: design and characterization," IEEE Trans. Appl. Supercond., vol. 21, no. 3, pp. 616-619, Jun. 2011.

[6] W. Shan, M. Takeda, T. Kojima, Y. Uzawa, S. Shi, T. Noguchi, and Z. Wang, "Low-noise waveguide-type NbN/AlN/NbN SIS mixers approaching terahertz frequencies," IEEE Trans. Microw. Theory Tech., vol. 58, no. 4, pp. 841-848, Apr. 2010.

[7] Y. Irimajiri, A. Kawakami, I. Morohashi, M. Kumagai, N. Sekine, S. Nagano, S. Ochiai, S. Tanaka, Y. Hanado, Y. Uzawa, and I. Hosako, "Development of a superconducting low-noise 3.1-THz hot electron bolometer receiver," IEEE Trans. THz Sci. Technol., vol. 5, no. 6, pp. 1154-1159, Nov. 2015.

[8] D. Buchel, P. Putz, K. Jacobs, M. Schultz, U. U. Graf, C. Risacher, H. Richter, O. Ricken, H.-W. Hubers, R. Gusten, C. E. Honingh, and J. Stutzki, "4.7-THz superconducting hot electron bolometer waveguide mixer," IEEE Trans. THz Sci. Technol., vol. 5, no. 2, pp. 207-214, Mar. 2015.

[9] I. Tretyakov, S. Ryabchun, M. Finkel, S. Maslennikov, A. Maslennikova, N. Kaurova, A. Lobastova, B. Voronov, and G. Gol'tsman, "Ultrawide noise bandwidth of $\mathrm{NbN}$ hot-electron bolometer mixers with in situ gold contacts," IEEE Trans. Appl. Supercond., vol. 21, no. 3, pp. 620-623, Jun. 2011.

[10] H. Shimakage, Y. Uzawa, M. Tonouchi, and Z. Wang, "Noise temperature measurement of YBCO Josephson mixers in millimetre and submillimeter waves," IEEE Trans. Appl. Supercond., vol. 7, no. 2, pp. 2595-2598, Jun. 1997.

[11] O. Harnack, M. Darula, J. Scherbel, J.-K. Heinsohn, M. Siegel, D. Diehl, and P. Zimmermann, "Optimization of a $115 \mathrm{GHz}$ waveguide mixer based on an HTS Josephson junction,” Supercond. Sci. Technol., vol. 12, no. 11, pp. 847-849, Nov. 1999.

[12] J. Scherbel, M. Darula, O. Harnack, and M. Siegel, "Noise properties of HTS Josephson mixers at $345 \mathrm{GHz}$ and operating temperatures at 20 K," IEEE Trans. Appl. Supercond., vol. 12, no. 2, pp. 1828-1831, Jun. 2002.

[13] M. Malnou, C. Feuillet-Palma, C. Ulysse, G. Faini, P. Febvre, M. Sirena, L. Olanier, J. Lesueur, and N. Bergeal, "High-Tc superconducting Josephson mixers for terahertz heterodyne detection,” J. Appl. Phys., vol. 116, no. 7, pp. 074505 074505-11, Aug. 2014.

[14] C. P. Foley, E. E. Mitchell, S. K. H. Lam, B. Sankrithyan, Y. M. Wilson, D. L. Tilbrook, and S. J. Morris, "Fabrication and characterisation of YBCO single grain boundary step edge junctions," IEEE Trans. Appl. Supercond., vol. 9, no. 2, pp. 4281-4284, Jun. 1999.

[15] T. Zhang, J. Du, Y. J. Guo, X. Sun, “A 7-8.5 GHz high-performance MMIC HTS Josephson mixer”, IEEE Microw. Wireless Compon. Lett., vol. 23, no. 8, pp. 427-429, Aug. 2013.

[16] J. Du, T. Zhang, Y. J. Guo, and X. Sun, "A high-temperature superconducting monolithic microwave integrated Josephson down-converter with high conversion efficiency," Appl. Phys. Lett., vol. 102, no. 21, pp. 212602 - 212602-4, May 2013.

[17] J. Du, J. Wang, T. Zhang, D. Bai, Y. J. Guo, and Y. He, "Demonstration of a portable HTS MMIC microwave receiver front-end," IEEE Trans. Appl. Supercond., vol. 25, no. 3, pp. 1500404, Jun. 2015.

[18] J. Du, A. D. Hellicar, K. E. Leslie, N. Nikolic, S. M. Hanham, J. C. Macfarlane, and C. P. Foley, "Towards large scale HTS Josephson detector arrays for THz imaging," Supercond. Sci. Technol., vol. 26, no. 11, pp. 115012 - 115012-7, Oct. 2013.

[19] J. Du, K. Smart, L. Li, K. E. Leslie, S. M. Hanham, D. H. C. Wang, C. P. Foley, F. Ji, X. D. Li, and D. Z. Zeng, “A cryogen-free HTS Josephson junction detector for terahertz imaging," Supercond. Sci. Technol., vol. 28, no. 8, pp. 084001 084001-7, Jul. 2015.

[20] P. F. Goldsmith, Quasioptical Systems: Gaussian Beam Quasioptical Propagation and Applications. New York, USA: IEEE Press, 1998.

[21] T. V. Duzer and C. W. Turner, Principles of Superconductive Devices and Circuits. Upper Saddle River, NJ, USA: Prentice-Hall, 1999.

[22] Http://www.vadiodes.com. 